\title{
Automatically Classifying Students in Need of Support by Detecting Changes in Programming Behaviour
}

\author{
Anthony Estey \\ University of Victoria \\ aestey@uvic.ca
}

\author{
Hieke Keuning \\ Open University of the \\ Netherlands and Windesheim \\ University of Applied Sciences \\ hw.keuning@windesheim.nl
}

\author{
Yvonne Coady \\ University of Victoria \\ ycoady@uvic.ca
}

\begin{abstract}
Educational research has established that learning can be defined as an enduring change in behaviour, which results from practice or other forms of experience. In introductory programming courses, proficiency is typically approximated through relatively small but frequent assignments and tests. Scaling these assessments to track significant behavioural change is challenging due to the subtle and complex metrics that must be collected from large student populations.

Based on a four-semester study, we present an analysis of learning tool interaction data collected from 514 students and 38,796 solutions to practice programming exercises. We first evaluate the effectiveness of measuring workflow patterns to detect students at-risk of failure within the first three weeks of the semester. Our early predictor analysis accurately detects $81 \%$ of the students who struggle throughout the course. However, our early predictor also captures transient struggling, as $43 \%$ of the students who ultimately did well in the course were classified as at-risk. In order to better differentiate sustained versus transient struggling, we further propose a trajectory metric which measures changes in programming behaviour. The trajectory metric detects $70 \%$ of the students who exhibit sustained struggling, and mis-classifies only $11 \%$ of students who go on to succeed in the course. Overall, our results show how detecting changes in programming behaviour can help us differentiate between learning and struggling in CS1.
\end{abstract}

\section{Keywords}

CS1; programming behaviour; educational data mining; predictors of success; automated classification techniques

\section{INTRODUCTION}

In recent years, there has been a spike in enrollment in computer science programs [22], but failure rates are still high [18]. As class sizes increase, it is extremely difficult for instructors to identify and support struggling students. In

Permission to make digital or hard copies of all or part of this work for personal or classroom use is granted without fee provided that copies are not made or distributed for profit or commercial advantage and that copies bear this notice and the full citation on the first page. Copyrights for components of this work owned by others than ACM must be honored. Abstracting with credit is permitted. To copy otherwise, or republish, to post on servers or to redistribute to lists, requires prior specific permission and/or a fee. Request permissions from permissions@ acm.org.

SIGCSE '17, March 08-11, 2017, Seattle, WA, USA

(C) 2017 ACM. ISBN 978-1-4503-4698-6/17/03 . \$ $\$ 15.00$

DOI: http://dx.doi.org/10.1145/3017680.3017790
CS1 courses, lecture content typically builds upon material covered in previous weeks, making it difficult for students who fall behind early to catch up on their own $[2,14]$.

Previous work on predictors of success in CS1 suggest that low-performing students, who tend not to recover, can be identified within the first few weeks of the semester $[1,5]$. Studies on debugging proficiency $[8,11,12,20]$ and study behaviour $[4,6,16]$ have also shown how patterns in log data can be used to accurately predict student performance.

Learning can be defined as an enduring change in behaviour, which results from practice or other forms of experience [15]. We build on the results of previous studies by investigating whether the inaccuracies found in early predictors of student performance can be reduced through an analysis of log data that measures changes in programming behaviour over time. If patterns associated with effective learning can be automatically classified in log data early in the semester, it may be possible to better guide students atrisk of failure towards more productive learning behaviour before it is too late. This motivates the key research question behind this study: Can analysis of patterns in interaction data help us understand how to detect and measure learning in CS1? To answer our key question, our analysis considers the following intermediate research questions:

RQ1: How accurately do early predictors of performance identify students who are unsuccessful in the course?

RQ2: How can early predictors be enhanced by a trajectory metric to classify behavioural change over time?

RQ3: How well can the trajectory metric be extended to evaluate differences in proficiency across topics?

This paper presents the results of a four-semester study on interaction data collected from 514 students who voluntarily used a programming practice tool. This study was designed to evaluate student learning by detecting changes in programming behaviour over time. The main contributions are (1) the introduction of metrics necessary to quantify learning through analysis of behavioural change; (2) insight into the issues associated with early at-risk student identification; and (3) a repository containing the tool and data used in this study to enable future reproduction studies [7].

Section 2 provides an overview of related work and background context. Section 3 presents our methodology, and then Section 4 overviews the results. In Section 5, we derive our analysis of the data and provide a discussion of our study in general. Finally, Section 6 concludes the paper and discusses critical paths for future work. 


\section{BACKGROUND AND RELATED WORK}

Our work builds upon a number of computer science education research domains, including educational data mining, interactive learning environments, and predictors of success.

Large-scale studies analyzing compilation and debugging behaviour $[3,8,9,11,12]$ provide evidence that log data can be used to accurately rank student debugging proficiency. Spacco et al. [16] use log data to identify "flailing" students, defined as those who are unlikely to solve a problem given an infinite amount of time. Yakymova et al. [21] classify learning strategies in CS1 as either surface-level or deep learning. Piech et al. [13] use a model to illustrate student progress through homework, with several "sink" states, where students exhibited a serious functional problem and were very unlikely to transition out of the state over multiple code updates. Edwards et al. [4] filter out students who consistently achieved high or low grades to focus on why students were successful in some activities, but not in others. Watson et al. [19] compare traditional and dynamic predictors of programming success, and warn against using metrics that fail to reflect changes in a student's learning progress over time. Our work builds upon these previous findings; our further contribution is a four-semester study where early predictors are combined with a metric used to identify behavioural change in the context of practice programming sessions using a learning tool.

Keuning et al. [10] provide a comprehensive review of available tools and the types of feedback they offer. VanLehn [17] compares electronic tutoring with human tutoring, and found the two to be equally effective for increasing learning in STEM topics. The learning tool used in our study provides students with hints to progressively lead them to a solution of each problem. The tool collects interaction data, which we analyze as a post-process to better understand how learning aligns with changes in programming behaviour.

\section{METHODOLOGY}

In a recent ITiCSE Working Group, Ihantola et al. [7] identified the critical need for validation and replication studies, to better understand contributing factors and reasons for results. From the taxonomy for replicating studies they propose, we begin with an 'extended analysis (A)' type of verification study, as it extends prior work by looking at a previously analyzed data set, but adds new analysis methods. To enable other researchers to do other types of reproduction studies, we provide a repository ${ }^{1}$ containing the open-source learning tool used for this study, the interaction data collected over the past 2 years, and a number of interactive visualizations based on our analysis.

\subsection{Data collection}

The data for this study was collected over four semesters from a 13-week CS1 course taught in Java. Topics covered include variables, control flow, methods, conditionals, loops, $\mathrm{I} / \mathrm{O}$, arrays, searching and sorting algorithms, and objects.

Interaction data was collected from a programming practice tool. Tool use was completely voluntary, and did not affect student grades in any way. Students used an embedded editor to work through exercises in a web-browser. The tool was introduced as a supplemental practice resource offering exercises similar to those presented during weekly labs. The

\footnotetext{
${ }^{1}$ www.github.com/aestey/phd
}

tool provided automatic feedback to students on submission correctness. Buttons to compile code, run code, submit a solution, get a hint, and ask a question were all instrumented to collect student interaction patterns. As an optional feature, students could use a series of hints that progressively lead them through each question, and the final hint provided a full sample solution. Our previous work [5] provides more detailed information about the features present in the learning tool, including the types of hints and exercises provided.

Data was collected from the 514 students who opted to take part in this study, out of the 718 enrolled in our course over the past four semesters. Log data was linked to final exam scores, which were standardized to account for potential changes in difficulty between semesters.

\subsection{Early predictors}

Our previous work found that, on average, high-performing students had higher compile rates and lower hint usage than students who failed [5]. In this study, we first examine how effective these metrics are as early predictors for identifying individual students who are at-risk of failing. This analysis considers data collected three weeks into the semester.

\subsection{Trajectory metrics}

We extend the early analysis to further explore whether early predictors can be refined by comparing changes between subsequent programming sessions. To detect students who struggle early in the semester, and continue to struggle over time, we introduce two metrics:

Baseline: the metric calculated to provide an initial measure of proficiency on each question attempted.

Trajectory: the metric calculated to quantify changes in programming behavior over subsequent attempts.

As students work through practice exercises, they are given a score between 0.0 and 1.0 based on their compile and hint usage. A score of 0.0 on a question denotes that only hints were used, and no code was compiled. A 0.25 means hints were used before any code compilations. A 0.5 value represents a comparable split between unassisted progress and hint usage. If progress was made with minimal support a 0.75 is awarded, and a score of 1.0 signifies that a student worked through the question correctly without using any hints. The value of the baseline metric ranges between 0.0 and 1.0, and is calculated by averaging the individual question scores for each student's first attempt on each question. Table 1 shows sample data for two students, and their associated baseline metric values.

Table 1: Baseline metric showing the number and order of hints and compiles for each question ( $Q \#)$

\begin{tabular}{|c|c|c|c|c|}
\hline & $Q \#$ & Behaviour & Score & Baseline \\
\hline \multirow{4}{*}{ Student A } & 1 & $\mathrm{CCC}$ & 1.0 & \multirow{4}{*}{0.88} \\
\hline & 2 & $\mathrm{CCCCC}$ & 1.0 & \\
\hline & 3 & ССССССССC & 1.0 & \\
\hline & 4 & СССНННСС & 0.5 & \\
\hline \multirow{3}{*}{ Student B } & 1 & HHHHH & 0.0 & \multirow{3}{*}{0.25} \\
\hline & 2 & $\mathrm{CCHC}$ & 0.5 & \\
\hline & 3 & НHНHCHH & 0.25 & \\
\hline
\end{tabular}

The trajectory score measures changes in programming behaviour across programming sessions. Sessions are determined by time stamps, and are delineated by periods of time when a student does not interact with the learning tool for more than two hours. Whereas the baseline metric 

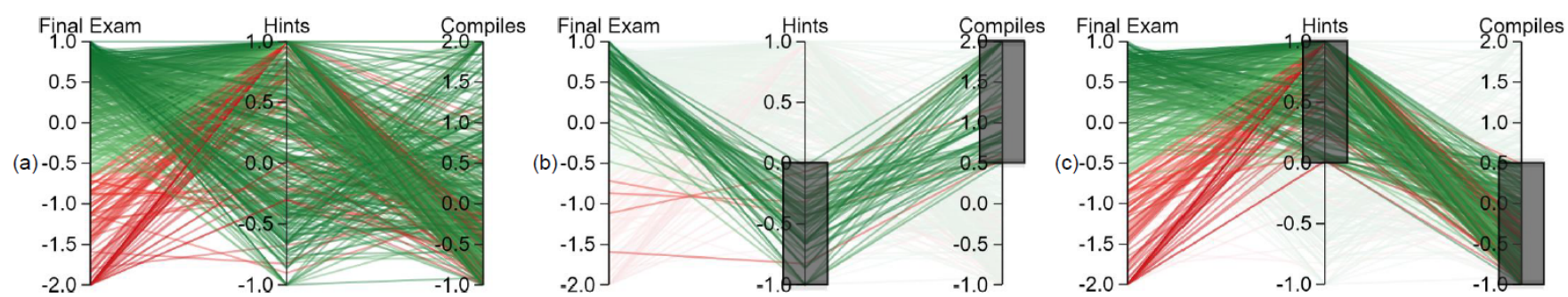

Figure 1: (a) Standardized final exam scores, hint usage, and compilation numbers for 514 students over four semesters. Students who passed the final exam are represented with green lines, red lines represent failure. (b) Students with below average hint usage and above average compile rates are selected, as highlighted by the rectangles on the axes. Among this group, only 4 students, represented by red lines, were unsuccessful on the final exam. (c) Selects students with above average hint usage and below average compile rates.

represents the average question score during each student's first attempt, the trajectory score represents the changes in scores between attempts. The trajectory score considers only data from exercises revisited when a correct solution was not submitted in previous attempts. When comparing individual scores between subsequent attempts, a value above 0.5 represents a change in behaviour towards unassisted progress, whereas below 0.5 signifies that hints were requested earlier or more often.

Unsuccessful students may not show signs of struggle until later in the semester, or may only struggle to progress through certain course material. We also apply our trajectory metrics to interaction data separated by topic, to identify at-risk behaviour related to a specific topic.

The metric calculations are not yet a feature included in the tool, and were completed as a post-process using interaction data collected by the tool.

\section{RESULTS}

First, we explore how well early predictors can be used to identify students in need of assistance. We then explore whether the accuracy of early predictors can be improved by measuring changes in learning behaviour over time with our trajectory metrics. Our results are presented through screen captures of the interactive parallel coordinates graphs found in our data repository, and are best viewed in colour.

\subsection{Early predictors (RQ1)}

Figures 1 and 2 show standardized compilation attempts, hint usage, and final exam scores for our student population. The graphs are colored based on exam performance, green representing passing grades and red failure. Figure 1a illustrates the variation across student data sets collected for the study. Our goal was to examine how well compile rates and

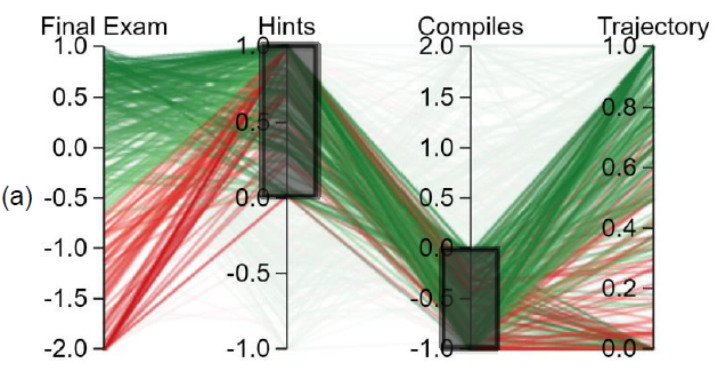

Figure 2: (a) Students identified as at-risk by the baseline metric, shown by the rectantangles selecting above average hint usage and below average compile rates. (b) Students who would be filtered from (a) by the trajectory metric, dropping the false positive rate from $43 \%$ to $11 \%$, and the true positive from $81 \%$ to $70 \%$. hint usage can be used to identify students struggling early in the semester. Figure 1 b selects only students with below average hint usage and above average compilation rates, as highlighted by the shaded rectangles on the axes. Only 4 of the 94 students who were unsuccessful on the final exam exhibited this behaviour. These 4 students represent the relatively small number of false negatives. Conversely, Figure 1c shows students with below average compilation rates and high hint usage, where 76 of the 94 (81\%) students who received a failing final exam grade are shown.

Figures $1 \mathrm{~b}$ and $1 \mathrm{c}$ suggest that, in general, high compile rates and low hint usage correlate with success. Hint and compile rate scores for each student positively and significantly correlate with midterm exam grades $(p<0.01)$, and also final exam grades $(\mathrm{p}<0.01)$. This does not mean, however, that these early predictors can be used to accurately identify only students who are genuinely at-risk of failing the final exam. At this point in the semester, it is very difficult to distinguish between the 257 students identified as at-risk; Figure 1c illustrates the wide range of final exam grades for these students. Table 4.1 shows that 181 of the 257 students appeared to be struggling early, but managed to succeed on the final exam without intervention. These 181 students make up $43 \%$ of all students who succeed in the course, a relatively high false positive rate.

Table 2: Early at-risk identification

\begin{tabular}{|l|c|c||c|}
\hline Classification: & At-risk & Not at-risk & Total \\
\hline Fail exam & 76 & 18 & 94 \\
\hline Pass exam & 181 & 239 & 420 \\
\hline
\end{tabular}

\subsection{Trajectory over time (RQ2)}

The trajectory metric considers data from exercises revisited when a correct solution was not submitted in previous attempts. Negative trajectory scores represent instances

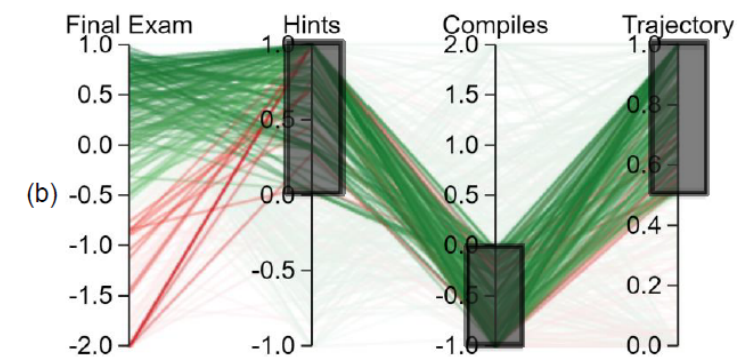



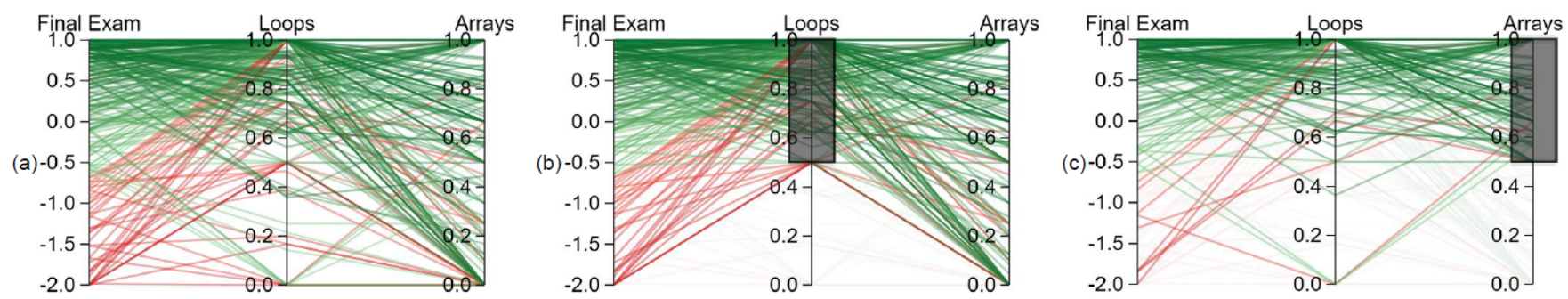

Figure 3: a) Standardized final exam grades along with loop and array trajectory scores for data collected 6 weeks into the course. b) Selects only students with positive trajectory scores on loop exercises. c) Selects only students with positive trajectory scores on arrays.

where students are actively struggling to complete an exercise over multiple subsequent sessions.

Figure 2a differs from Figure 1c in that an additional axis is added representing trajectory scores. Figure 2a shows the wide range of final exam outcomes achieved by the $257 \mathrm{stu}-$ dents considered in the trajectory metric analysis. Applying the trajectory metric filters out 134 false-positives, reducing the number from $181(43 \%)$ to $47(11 \%)$, while true positives dropped from $76(81 \%)$ to $63(70 \%)$. The students who would be filtered out through trajectory analysis are shown in Figure 2b. Overall, using the trajectory identifies $70 \%$ of the students who failed, while $11 \%$ are mis-classified as at-risk. Table 3 summarizes overall trajectory results.

Applying the trajectory metrics demonstrated precisely how the accuracy of the early results can be improved by considering changes in behaviour across programming sessions. The trajectory metric analysed the population of students identified by early analysis as potentially at-risk, and was used to more accurately identify students who were genuinely at-risk. Among this group, the trajectory score positively and significantly correlates with midterm exam grades $(p=0.02)$, and final exam grades $(p<0.01)$.

The trajectory metric results reveal that many students exhibit quantifiable changes in behaviour over time. However, it is entirely possible that students we claim to be mis-classified were identified accurately based on their behaviour at the time of the analysis. As there were a limited number of topics available in the programming tool at this point in the semester, the next step in the study explores if unsuccessful students who were still mis-classified could be properly identified based on their interaction data collected from later topics.

Table 3: Identification after trajectory filter

\begin{tabular}{|l|c|c||c|}
\hline Classification: & At-risk & Not at-risk & Total \\
\hline Fail exam & 63 & 31 & 94 \\
\hline Pass exam & 47 & 373 & 420 \\
\hline
\end{tabular}

\subsection{Trajectory on a topic basis (RQ3)}

To measure changes in programming behaviour across topics, an analysis was performed on data collected at the end of the sixth week of the course. At this point, loops and arrays were two of the available topics in the tool. For the 223 students who completed and revisited exercises on both topics, Figures 3 and 4 show final exam grades along with scores generated by our trajectory metric applied within each topic.

Figure 3a shows the entire population of participants in this part of the study, whereas Figure $3 \mathrm{~b}$ selects only students with positive trajectory scores on loop exercises. Of the students who ended up passing the course, 172 (95\%) of them have positive trajectory scores on loops exercises compared to $31(78 \%)$ of students who failed the course. Figure $3 \mathrm{c}$ selects students with positive trajectory scores on array exercises. At this point in the course, 125 (69\%) students who passed the course exhibit this behaviour, compared to only 9 (23\%) students who failed the course. Table 4.3 summarizes these results.

Figure $4 \mathrm{~b}$ and Figure 4c illustrate how productivity levels may be different between topics. Figure $4 \mathrm{~b}$ selects students with positive trajectory scores on both loops and arrays. Among students who pass, 122 (67\%) exhibit this behaviour, compared to only 8 (20\%) failing students. Figure 4c selects students with positive trajectory scores on loop exercises, and negative scores on arrays. Figure $4 \mathrm{c}$ illustrates how $25(63 \%)$ of the students who ended up failing the course transition from a positive trajectory score on loop exercises to a negative productivity score on array exercises.

The results illustrate distinguishable changes in behaviour across topics, especially among the students who eventually fail the course. Among this group, the percentage of students identified as at-risk grows by $57 \%$ when comparing interaction data collected on loop exercises to data collected on array exercises. This illustrates that, in addition to applying trajectory metrics to measure changes in behaviour over
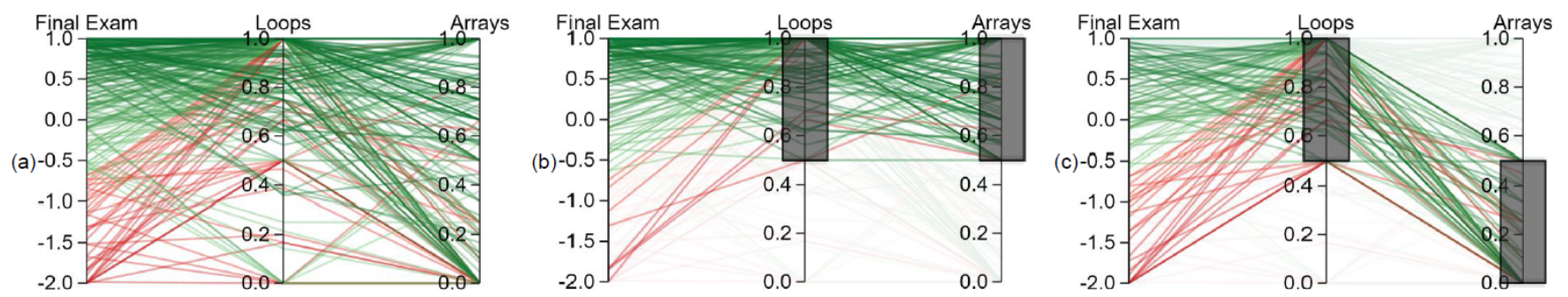

Figure 4: (a) The full data set without any filters applied. (b) Selects students with positive trajectory scores on both loop and array exercises. c) Selects students with positive loop scores and negative array scores. Of the 40 students who failed the course in this data set, $63 \%$ students exhibit these changes in behaviour between topic areas introduced two weeks apart. 
time within a single topic, measuring behavioural changes across multiple topics may also reveal students struggling in very specific areas of the course.

Table 4: Trajectory metric across topic areas

\begin{tabular}{|l|c|c|c|c|}
\hline & \multicolumn{2}{|c|}{ Loops } & \multicolumn{2}{c|}{ Arrays } \\
Classification: & At-risk & Not at-risk & At-risk & Not at-risk \\
\hline Fail exam (40) & 9 & 31 & 31 & 9 \\
\hline Pass exam (182) & 10 & 172 & 57 & 125 \\
\hline
\end{tabular}

\section{ANALYSIS AND DISCUSSION}

In this work, we explored whether behaviour patterns can be found in interaction data that allow for dynamic classification of learning in CS1. Our results support the findings of previous studies that suggest that a large number of atrisk students can be identified early in the semester. On the other hand, our new approach using the trajectory metric highlights the fact that many students struggle at different times, and in different topics, throughout the semester.

It is important that we continue to improve the efficacy of early predictors in CS1; struggling students need to be identified and supported as early as possible in the semester. It is also important to acknowledge that there may not be a single metric that can accurately predict the outcome of every student at one particular time, simply because learning takes place over time. Although our early predictor correlates with final exam performance, the correlation strength was increased by a relatively large group of students who were able to consistently work through practice exercises without assistance and also achieve very high exam grades. Previous work suggests that many of these students may have entered the course with previous programming experience, which may have contributed to the fact that we did not measure significant behavioural changes for them. This motivates our key research question: can analysis of patterns in interaction data help us understand how to detect and measure learning in CS1?

There was significantly more noise, variation, and change over time found in the data collected from the remaining students included in the study. The variation made it very difficult to distinguish between the large number of students initially identified as at-risk. This illustrates why a static predictor that correlates with course performance does not necessarily mean it can accurately detect at-risk students. We incorporated a trajectory metric into our analysis because the students we want to identify and support are not necessarily the students who enter the course without strong programming ability, but those who remain unable to demonstrate progress without assistance.

Our results reported on how the trajectory metric was used to increase the accuracy of early predictors in identifying at-risk students, but we made no mention about how our metrics could be used to identify learning. In our study, early predictors classified 257 students as at-risk based on their programming behaviour over the first three weeks of the semester. Among this group, the trajectory metric identified 134 students who showed productive changes in programming behaviour over time. If learning can be defined as an enduring change in behaviour, it can be argued that our analysis reveals that these 134 students demonstrated learning. These 134 students make up $52 \%$ of the 257 students identified by our early predictor analysis as at-risk. If we can better understand the reasons why these students transition from at-risk behaviour to behaviour associated with success, it may serve as a model from which to base intervention efforts aimed at supporting struggling students.

Our study differs from previous works that analysed early predictors in CS1 because we incorporate metrics that measure behavioural change over time. In our study, these metrics were used to improve the accuracy of early predictors, but measuring learning is not limited to our specific metrics. Although in our study we created metrics to detect highlevel changes in programming behaviour, the same general metrics can be applied to similar studies focusing on other factors that contribute to success in CS1, assuming the data collected allows for the following:

- Data points to generate a quantifiable measure.

- Capability to identify each student individually.

- Time stamps to evaluate change over time.

- Data recorded on the same or very similar exercises to compare between students and analyze progress.

These provide a guideline for what we found was necessary to measure behaviour in CS1, but our metrics can be extended and improved in future work. It is important to recognize the limitations and threats to validity of our study. Students enter our course with a wide range of previous experience, and the factors that may contribute to student success outside of programming behaviour are not considered in this study. Interaction data would be affected for students who studied in groups. Also, the analysis includes only the data from students who both chose to use the practice tool and also consented to take part in the study.

Our analysis metrics penalize hint usage, but students may not view hints only when struggling to solve an exercise. A student may exhibit different behaviour when learning a new concept or preparing for an exam, but we did not incorporate any session-specific behaviours into our metrics. The analysis metrics also do not take question difficulty into account. For the baseline metric, if a student was to skip through easy questions in a topic, and was then unable to progress through a difficult exercise without hints, this behaviour would result in a lower score than successfully completing trivial exercises without assistance. Similarly, for the trajectory metric, it may be important to know if a student is revisiting and struggling to complete an introductory question or a question designed to be challenging.

Despite these limitations, the results of this preliminary study using trajectory metrics are encouraging, and it is our hope that our findings measuring changes in behaviour might inspire other researchers to incorporate metrics that consider behavioural change into their own analysis.

\section{CONCLUSIONS AND FUTURE WORK}

This study presents an analysis of interaction data collected from 514 students working on exercises in a programming practice tool, to help us understand how to detect and measure learning in CS1. We first explore early predictors of at-risk behaviour, after which we propose two metrics, baseline and trajectory, that we use to quantify changes in student behaviour over time and in different course topics. We show that these metrics are better at differentiating between sustained versus transient struggling.

We identify and describe several issues that arise when trying to identify struggling students. In future work, we plan to investigate whether interaction data can help us apply a difficulty rating to each exercise. Then, we can eval- 
uate whether our analysis metrics can be improved by factoring question difficulty into the analysis. We also suggest collecting information on the objectives of the students during each session, as this information may provide additional context allowing educators to better understand the factors contributing to measured outcomes in their analysis.

We are also interested in further analysing the interaction data of students who did not show productive changes in behaviour. Our assumption is that students who are actively working through ungraded practice exercises are highly invested in learning. Unfortunately, 63 students in our study exhibited patterns associated with sustained struggle. Further analysis may reveal patterns among these students that can be generalized as impediments to success in CS1.

In our following work, we plan to further explore the interaction data of the students initially identified as at risk in our study. Further analysis using different metrics that compares the interaction data of students who show changes in behaviour with those who do not may reveal interesting patterns related to the learning process. Once we better understand the factors contributing to behavioural change, we can begin providing supportive intervention aimed at guiding struggling students towards productive behaviour.

\section{REFERENCES}

[1] A. Ahadi, R. Lister, H. Haapala, and A. Vihavainen. Exploring machine learning methods to automatically identify students in need of assistance. In Proc. of the Conference on International Computing Education Research (ICER), pages 121-130, 2015.

[2] A. Ahadi, R. Lister, and D. Teague. Falling behind early and staying behind when learning to program. In Psychology of Programming (PPIG), 2014.

[3] A. Altadmri and N. C. Brown. 37 million compilations: Investigating novice programming mistakes in large-scale student data. In Proc. of the ACM Technical Symposium on Computer Science Education (SIGCSE), pages 522-527, 2015.

[4] S. H. Edwards, J. Snyder, M. A. Pérez-Quiñones, A. Allevato, D. Kim, and B. Tretola. Comparing effective and ineffective behaviors of student programmers. In Proc. of the Workshop on Computing Education Research (ICER), pages 3-14, 2009.

[5] A. Estey and Y. Coady. Can interaction patterns with supplemental study tools predict outcomes in cs1? In Proc. of the ACM Conference on Innovation and Technology in Computer Science Education (ITiCSE), pages 236-241, 2016.

[6] N. J. Falkner and K. E. Falkner. A fast measure for identifying at-risk students in computer science. In Proc. of the Conference on International Computing Education Research (ICER), pages 55-62, 2012.

[7] P. Ihantola, A. Vihavainen, A. Ahadi, M. Butler, J. Börstler, S. H. Edwards, E. Isohanni, A. Korhonen, A. Petersen, K. Rivers, M. A. Rubio, J. Sheard, B. Skupas, J. Spacco, C. Szabo, and D. Toll. Educational data mining and learning analytics in programming: Literature review and case studies. In Proc. of the 2015 ITiCSE on Working Group Reports, pages 41-63, 2015.

[8] M. C. Jadud. Methods and tools for exploring novice compilation behaviour. In Proc. of International
Workshop on Computing Education Research (ICER), pages 73-84, 2006.

[9] M. C. Jadud and B. Dorn. Aggregate compilation behavior: Findings and implications from 27,698 users. In Proc. of the Eleventh Annual International Conference on International Computing Education Research, ICER '15, pages 131-139, 2015.

[10] H. Keuning, J. Jeuring, and B. Heeren. Towards a systematic review of automated feedback generation for programming exercises. In Proc. of the Conference on Innovation and Technology in Computer Science Education (ITiCSE), pages 41-46, 2016.

[11] J. P. Munson and E. A. Schilling. Analyzing novice programmers' response to compiler error messages. $J$. Comput. Sci. Coll., 31(3):53-61, 2016.

[12] C. Murphy, G. Kaiser, K. Loveland, and S. Hasan. Retina: Helping students and instructors based on observed programming activities. In Proc. of the 40th ACM Technical Symposium on Computer Science Education, SIGCSE '09, pages 178-182, 2009.

[13] C. Piech, J. Bassen, J. Huang, S. Ganguli, M. Sahami, L. Guibas, and J. Sohl-Dickstein. Deep knowledge tracing. In Proc. of the Conference on Neural Information Processing Systems (NIPS), pages 505-513, 2015.

[14] A. Robins. Learning edge momentum: a new account of outcomes in cs1. Computer Science Education, 20(1):37-71, 2010.

[15] D. H. Schunk. Learning theories. Prentice Hall Inc., New Jersey, 1996.

[16] J. Spacco, P. Denny, B. Richards, D. Babcock, D. Hovemeyer, J. Moscola, and R. Duvall. Analyzing student work patterns using programming exercise data. In Proc. of the 46th ACM Technical Symposium on Computer Science Education, SIGCSE '15, pages 18-23, 2015.

[17] K. VanLehn. The relative effectiveness of human tutoring, intelligent tutoring systems, and other tutoring systems. Educational Psychologist, 46(4):197-221, 2011.

[18] C. Watson and F. W. Li. Failure rates in introductory programming revisited. In Proc. of the Conference on Innovation and Technology in Computer Science Education (ITiCSE), pages 39-44, 2014.

[19] C. Watson, F. W. Li, and J. L. Godwin. No tests required: Comparing traditional and dynamic predictors of programming success. In Proc. of the ACM Technical Symposium on Computer Science Education (SIGCSE), pages 469-474, New York, NY, USA, 2014. ACM.

[20] C. Watson, F. W. B. Li, and J. L. Godwin. Predicting performance in an introductory programming course by logging and analyzing student programming behavior. In Proc. of the IEEE International Conference on Advanced Learning Technologies (ICALT), pages 319-323, 2013.

[21] H. Yakymova, Y. Monteiro, and D. Zingaro. Study strategies and exam grades in cs1. In Proc. of the Western Canadian Conference on Computing Education (WCCCE), pages 24:1-24:3, 2016.

[22] S. Zweben and B. Bizot. 2015 Taulbee Survey. Computing Research News, 28(5), 2016. 